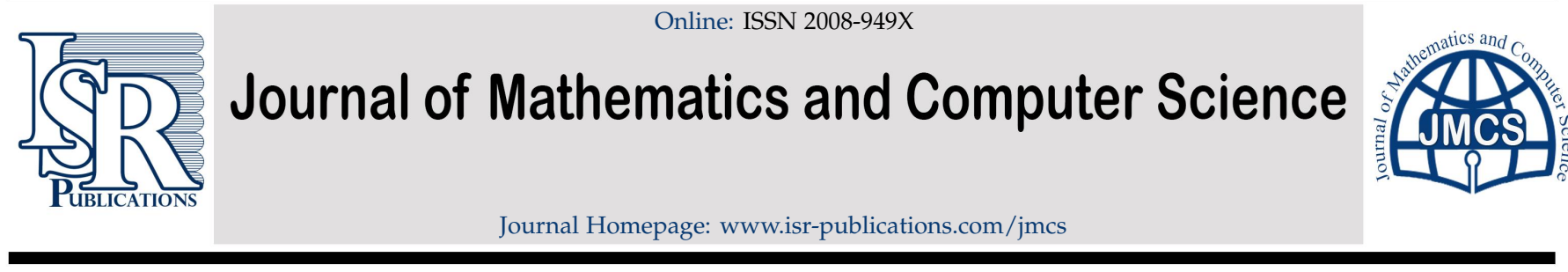

\title{
On Hyers-Ulam-Rassias stability of fractional differential equations with Caputo derivative
}

\author{
El-sayed El-hady ${ }^{a, b, *}$, Süleyman Öğrekçic \\ ${ }^{a}$ Mathematics Department, College of Science, Jouf University, P.O. Box: 2014, Sakaka, Saudi Arabia. \\ ${ }^{b}$ Basic Science Department, Faculty of Computers and Informatics, Suez Canal University, Ismailia, 41522, Egypt. \\ ${ }^{c}$ Department of Mathematics, Faculty of Arts and Science, Amasya University, Amasya, Turkey.
}

\begin{abstract}
In this article, we study the stability problem of some fractional differential equations in the sense of Hyers-Ulam and Hyers-Ulam-Rassias based on some fixed point techniques. In this way, we improve and generalize some recent results by dropping some basic assumptions.
\end{abstract}

Keywords: Hyers-Ulam stability, fractional differential equation, fixed point theory.

2020 MSC: 34A08, 37C25.

(C)2021 All rights reserved.

\section{Introduction}

The legendary question that has been posed by Ulam in 1940 ignited the theory of stability of functional (differential, difference, integral) equations. Ulam posed his question in the famous talk at the university of Wisconsin (see, e.g., [9, 13, 16, 18, 28, 31] for more details). The mentioned open problem can be stated as follows:

Let $G$ be a group and $\left(G^{*}, \rho\right)$ a metric group. Given $\varepsilon>0$, does there exist $\delta>0$ such that if $f: G \rightarrow G^{*}$ satisfies

$$
\rho(f(x y), f(x) f(y))<\delta
$$

for all $x, y \in G$, then a homomorphism $g: G \rightarrow G^{*}$ exists such that

$$
\rho(f(x), g(x))<\varepsilon, \forall x, y \in G ?
$$

Many well-known mathematicians have addressed Ulam's problem. For example, in 1941, Hyers introduced an affirmative answer in the special case where $G, G^{*}$ are Banach spaces (see [17]). Since then the problem is called Hyers-Ulam or Ulam-Hyers stability problem. In 1950, Aoki (see [3]) investigated this problem for additive mappings (see also [8]). A new name (Hyers-Ulam-Rassias) of the problem has been presented when Th. M. Rassias (see [27]) generalized the theorem of Hyers by considering the stability in the case of unbounded Cauchy differences. The interesting result introduced by M. Rassias reads as follows (see [27]).

\footnotetext{
*Corresponding author

Email address: elsayed_elhady@ci.suez.edu.eg (El-sayed El-hady)

doi: $10.22436 /$ jmcs.022.04.02
}

Received: 2020-06-17 Revised: 2020-07-22 Accepted: 2020-07-23 
Theorem 1.1. Consider $\mathrm{B}_{1}, \mathrm{~B}_{2}$ to be two Banach spaces, and let $\mathrm{f}: \mathrm{B}_{1} \rightarrow \mathrm{B}_{2}$ be a mapping such that the function $\mathrm{t} \mapsto \mathrm{f}(\mathrm{t} x)$ from $\mathbb{R}$ into $\mathrm{B}_{2}$ is continuous for each fixed $\mathrm{x} \in \mathrm{B}_{1}$. Assume that there exists $\theta \geqslant 0$ and $\mathrm{p} \in[0,1)$ such that

$$
\|f(x+y)-f(x)-f(y)\| \leqslant \theta\left(\|x\|^{p}+\|y\|^{p}\right), x, y \in B_{1} \backslash\{0\} .
$$

Then there exists a unique solution $\mathrm{T}: \mathrm{B}_{1} \rightarrow \mathrm{B}_{2}$ of the Cauchy equation with

$$
\|f(x)-T(x)\| \leqslant \frac{2 \theta\|x\|^{p}}{\left|2-2^{p}\right|^{\prime}}, x \in B_{1} \backslash\{0\} .
$$

The results of Hyers and Rassias have been generalized in many directions to other settings. For instance, several authors have studied the stability for differential equations (see $[2,19,21,23,24])$.

In general terms, stability has been studied using each of the following methods. The method Hyers put forward quite frequently called the direct one (see [17]), the method of invariant means (introduced in [29]), the fixed point method (see [4, 12]), the method based on sandwich theorems [26], and the method using the concept of shadowing (see [30]). The fixed point method is well-known as the second most popular method in proving the stability of functional equations. According to the best of our knowledge, Baker in 1991 (see [4]) was the first to use the fixed-point approach in the investigations of stability of functional equations. Basically, Baker employed some version of Banach fixed point theorem to obtain the Hyers-Ulam stability of functional equations.

It should be remarked that Diaz and Margolis in [15] proved a theorem of the alternative for any contraction mapping on a generalized complete metric space. The approach presented by Diaz and Margolis is widely used in different contexts, see, e.g., [1]. Fixed point techniques have been used in the literature in the investigation of Hyers-Ulam stability. For instance, in 2012, Ciepliński (see [14]) wrote a survey in which he presented some applications of various fixed-point theorems to the theory of the Hyers-Ulam stability of functional equations. In 2011, Brzdęk (see [10]) proved a fixed-point theorem for (not necessarily) linear operators and used it to obtain Hyers-Ulam stability results for a class of functional equations. Brzdęk and Ciepliński in [11] proved a fixed-point result in complete non-Archimedean metric spaces as well as in complete metric spaces.

In a recent paper, Jung in [21] used the fixed point approach to prove the stability of ceratin first order differential equations. In [5] Başci et al. studied the stability of some differential equations in the sense of Hyers-Ulam. In [7] the authors investigated the Hyers-Ulam stability for fractional differential equations including the new Caputo-Fabrizio fractional derivative. In [6] the Hyers-Ulam-Rassias stability for AbelRiccati-type first-order differential equations has been investigated and in [25] the author studied the stability of delay differential equations in the sense of Hyers-Ulam on unbounded intervals.

The goal of this paper is to investigate the Hyers-Ulam stability (HUS) and the Hyers-Ulam-Rassias stability (HURS) of some fractional differential equations. The main tool used in our analysis is the fixed point theory that has been introduced by Diaz and Margolis (see [15]). In our study, we drop some of the assumptions in some recent results. The rest of the article is organized as follows. In Section 2, we recall and discuss some basic definitions and lemmas that are necessary in the analysis. We also present the fixed point theorem that is our basic tool in the analysis. In Section 3, we will apply the fixed point method to study the stability the differential equation of interest, and in the last section, we conclude our work.

\section{Preliminaries}

Throughout the article, we will use $\mathbb{R}$ to denote the set of reals, $\mathbb{R}_{+}$to denote the set of positive reals, and $\mathbb{C}$ to denote the set of complex numbers. We first recall some definitions.

Definition 2.1. Given an interval $[a, b]$ of $\mathbb{R}$, the fractional order integral of a function $h \in \mathrm{L}^{1}([0, \mathrm{~b}], \mathbb{R})$ of order $\alpha \in \mathbb{R}_{+}$is defined by

$$
I_{a_{+}}^{\alpha} h(t)=\frac{1}{\Gamma(\alpha)} \int_{a}^{t}(t-s)^{\alpha-1} h(s) d s,
$$


where $\Gamma(\cdot)$ is the gamma function.

Definition 2.2. For a function $\mathrm{h}$ given on the interval $[\mathrm{a}, \mathrm{b}]$, the $\alpha$-th order Riemann-Liouville fractional derivative of $\mathrm{h}$, is defined by

$$
\left(D_{a_{+}}^{\alpha} h\right)(t)=\frac{1}{\Gamma(n-\alpha)}\left(\frac{d}{d t}\right)^{n} \int_{a}^{t}(t-s)^{n-\alpha-1} h(s) d s,
$$

where $n=[\alpha]+1$ and $[\alpha]$ denotes the integer part of $\alpha$.

Definition 2.3. For a function $\mathrm{h}$ given on the interval $[\mathrm{a}, \mathrm{b}]$, the Caputo fractional order derivative of $\mathrm{h}$, is defined by

$$
\left({ }^{c} D_{a_{+}}^{\alpha} h\right)(t)=\frac{1}{\Gamma(n-\alpha)} \int_{a}^{t}(t-s)^{n-\alpha-1} h^{(n)}(s) d s,
$$

where $n=[\alpha]+1$.

The following lemmas are well known, see, e.g., [22].

Lemma 2.4. For all $\mathrm{b}>0$ and $\mathrm{a}>-1$

$$
\int_{0}^{t}(t-s)^{b-1} s^{a} d s=\frac{\Gamma(b) \Gamma(a+1)}{\Gamma(b+a+1)} t^{b+a} .
$$

Lemma 2.5. For all $\lambda, v, w>0$, and for any $\mathrm{t}>0$, we have

$$
t^{1-v} \int_{0}^{t}(t-s)^{v-1} s^{\lambda-1} e^{-w s} d s \leqslant \max \left\{1,2^{1-v}\right\} \Gamma(\lambda)\left(1+\frac{\lambda}{v}\right) w^{-\lambda} .
$$

For a nonempty set $\mathrm{Y}$; we recall the concept of generalized metric on $\mathrm{Y}$ as follows.

Definition 2.6. A mapping $\rho: \mathrm{Y} \times \mathrm{Y} \rightarrow[0, \infty]$ is called a generalized metric on set $\mathrm{Y}$ if $\rho$ satisfies:

(G1) $\rho(x, y)=0$ if and only if $x=y$;

(G2) $\rho(x, y)=\rho(y, x)$ for all $x, y \in Y$;

(G3) $\rho(x, z) \leqslant \rho(x, y)+\rho(y, z)$ for all $x, y, z \in Y$.

Definition 2.7. The fractional differential equation

$$
F\left(f, y, D^{\alpha_{1}} y, \ldots, D^{\alpha_{n}} y\right)=0
$$

is said to be Hyers-Ulam stable if for a given $\varepsilon>0$ and a function $y$ which satisfies the inequality

$$
\left|F\left(f, y, D^{\alpha_{1}} y, \ldots, D^{\alpha_{n}} y\right)\right| \leqslant \varepsilon,
$$

there exists a solution $\mathrm{y}_{\alpha}$ of (2.1) such that $\left|\mathrm{y}(\mathrm{x})-\mathrm{y}_{\alpha}(\mathrm{x})\right| \leqslant \mathrm{K}_{1}(\varepsilon)$ and $\lim _{\varepsilon \rightarrow 0} \mathrm{~K}_{1}(\varepsilon)=0$. If the expression holds when we replace $\varepsilon$ and $\mathrm{K}_{1}(\varepsilon)$ by $\mathrm{f}(\mathrm{x})$ and $\mathrm{g}(\mathrm{x})$, where $\mathrm{f}, \mathrm{g}$ are appropriate functions not depending on $\mathrm{y}$ and $\mathrm{y}_{\alpha}$, then we say that the fractional differential equation has the generalized Hyers-Ulam stability, or the equation is Hyers-Ulam-Rassis stable.

In the following theorem, we introduce one of the fundamental results of fixed point theory. For the proof, we refer to [15]. This theorem will play an important role in proving our main results.

Theorem 2.8. Let $(\mathrm{Y}, \rho)$ be a generalized complete metric space. Assume that $\wedge: \mathrm{Y} \rightarrow \mathrm{Y}$ is a strictly contractive operator with $\mathrm{L}<1$, where $\mathrm{L}$ is a Lipschitz constant. If there exists a nonnegative integer $\mathrm{k}$ such that $\rho\left(\wedge^{k+1} y, \wedge^{k} y\right)<\infty$ for some $y \in Y$, then the following are true: 
(a) the sequence $\Lambda^{\mathrm{n}} \mathrm{y}$ converges to a fixed point $\mathrm{y}^{*}$ of $\Lambda$;

(b) $\mathrm{y}^{*}$ is the unique fixed point of $\Lambda$ in $\mathrm{Y}^{*}:=\left\{\mathrm{y}_{1} \in \mathrm{Y}: \rho\left(\Lambda^{\mathrm{k}} \mathrm{y}, \mathrm{y}_{1}\right)<\infty\right\}$;

(c) if $y_{1} \in Y^{*}$, then $\rho\left(y_{1}, y^{*}\right) \leqslant \frac{1}{1-L} \rho\left(\Lambda y_{1}, y_{1}\right)$.

In this article, by using Theorem 2.8, we study the Hyers-Ulam-Rassias stability and the Hyers-Ulam stability of the following fractional differential equation

$$
{ }^{c} D_{a_{+}}^{\alpha} y(x)=f(x, y(x)),
$$

where ${ }^{c} D_{a_{+}}^{\alpha}$ is the Caputo fractional derivative of order $\alpha \in(0,1)$ with the lower limit $a \in \mathbb{R}$, the unknown function $y$ is some continuous function, $y: I \rightarrow Y$ for some interval $I:[a, b]$ and a normed space $Y$.

\section{Main results}

In this section, we fix an interval $I:=[0, r]$ for some real number $r>0$, further, we define the set $S$ of all continuous functions on I by

$$
S:=\{f: I \rightarrow \mathbb{R} \mid f \text { is continuous }\}=C(I, \mathbb{R}) .
$$

The following result will be used in our analysis:

Lemma 3.1 ([5]). Define the function $\mathrm{d}: \mathrm{S} \times \mathrm{S} \rightarrow[0, \infty]$ with

$$
d(f, g):=\inf \left\{C \in[0, \infty]:|f(t)-g(t)| e^{-A t} \leqslant C \phi(t), t \in I\right\},
$$

where $\mathrm{A}>0$ is a given constant and $\phi: \mathrm{I} \rightarrow(0, \infty)$ is a given continuous function. Then $(\mathrm{S}, \mathrm{d})$ is a generalized complete metric space.

The following theorem is the main theorem of this article. It presents stability results of the fractional differential equation (2.2) in the sense of Hyers-Ulam-Rassias.

Theorem 3.2. Suppose that $f: I \times \mathbb{R} \rightarrow \mathbb{R}$ is some continuous function that satisfies the following Lipschitz condition

$$
\left|f\left(t, y_{1}\right)-f\left(t, y_{2}\right)\right| \leqslant L\left|y_{1}-y_{2}\right|
$$

for all $\mathrm{t} \in \mathrm{I}, \mathrm{y}_{1}, \mathrm{y}_{2} \in \mathbb{R}$, and a Lipschitz condition $\mathrm{L}>0$. Furthermore, if a continuously differentiable function $y: I \rightarrow \mathbb{R}$ satisfies

$$
\left|{ }^{c} D_{a_{+}}^{\alpha} y(x)-f(x, y(x))\right| \leqslant \varphi(t)
$$

for all $\mathrm{t} \in \mathrm{I}$ and a continuous and non-decreasing function $\varphi: \mathrm{I} \rightarrow(0, \infty)$, then there exists a unique solution $\mathrm{y}_{0}$ of (2.2) such that

$$
\left|y(t)-y_{0}(t)\right| \leqslant \frac{N}{\Gamma(\alpha) A-N L} \varphi(t)
$$

for all $\mathrm{t} \in \mathrm{I}$ and arbitrary constants $\mathrm{A}$ and $\mathrm{N}$ such that the inequalities

$$
\frac{\mathrm{NL}}{\mathrm{A} \Gamma(\alpha)}<1, \quad \max \left\{1,2^{1-\alpha}\right\}\left(\frac{\alpha+1}{\alpha}\right) \leqslant \mathrm{N} e^{A t}
$$

hold.

Proof. First, we consider the set $S$ defined by (3.1) and define the following function $d: S \times S \rightarrow[0, \infty]$ with

$$
d(f, g)=\inf \left\{C \in[0, \infty]:|f(t)-g(t)| e^{-A t} \leqslant C \varphi(t), t \in I\right\} .
$$

In view of Lemma 3.1, $(S, d)$ is a complete generalized metric space (see also, e.g., [20]). Now, define the 
operator $\mathcal{T}: S \rightarrow S$ by

$$
(\mathcal{T} y)(x)=y(0)+\frac{1}{\Gamma(\alpha)} \int_{0}^{t}(t-s)^{\alpha-1} f(s, y(s)) d s, t \in I
$$

for all $y \in S$. It should be noted that $\mathcal{T}$ is well-defined since both $f$ and $F$ are continuous.

Next, we will check that the operator $\mathcal{T}$ is strictly contractive on the set $S$. Note first that using Lemma 2.5 with $\alpha=v, t_{0}=0, \lambda=1, w=A$ we get

$$
\int_{0}^{t}(t-s)^{\alpha-1} e^{A s} d s \leqslant \frac{1}{A} \max \left\{1,2^{1-\alpha}\right\}\left(1+\frac{1}{\alpha}\right) t^{\alpha-1} .
$$

Using the above inequality we write

$$
\begin{aligned}
\int_{0}^{t}(t-s)^{\alpha-1} \varphi(s) e^{A s} d s & \leqslant \varphi(t) \int_{0}^{t}(t-s)^{\alpha-1} e^{A s} d s \\
& \leqslant \max \left\{1,2^{1-\alpha}\right\}\left(\frac{1+\alpha}{\alpha}\right) t^{\alpha-1} \frac{\varphi(t)}{A} \leqslant \frac{N}{A} \varphi(t) e^{A t},
\end{aligned}
$$

where $\mathrm{N}$ is some constant such that the inequality

$$
\max \left\{1,2^{1-\alpha}\right\}\left(\frac{1+\alpha}{\alpha}\right) \leqslant N e^{A t}
$$

holds. Now, for any $h_{1}, h_{2} \in S$, let $C_{h_{1}, h_{2}} \in[0, \infty]$ be an arbitrary constant with $d\left(h_{1}, h_{2}\right) \leqslant C_{h_{1}, h_{2}}$ that is

$$
\left|h_{1}(t)-h_{2}(t)\right| e^{-A t} \leqslant C_{h_{1}, h_{2}} \varphi(t)
$$

for all $t \in I$. Then by using (3.4), (3.5), and (3.6), it follows that for $h_{1}, h_{2} \in S$,

$$
\begin{aligned}
\left|\mathcal{T h}(t)-\mathcal{T h} h_{2}(t)\right| & \leqslant\left|\frac{1}{\Gamma(\alpha)} \int_{0}^{t}(t-s)^{\alpha-1}\left\{f\left(s, h_{1}(s)\right)-f\left(s, h_{2}(s)\right)\right\} d s\right| \\
& \leqslant \frac{1}{\Gamma(\alpha)} \int_{0}^{t}(t-s)^{\alpha-1}\left|f\left(s, h_{1}(s)\right)-f\left(s, h_{2}(s)\right)\right| d s \\
& \leqslant \frac{L}{\Gamma(\alpha)} \int_{0}^{t}(t-s)^{\alpha-1}\left|h_{1}(s)-h_{2}(s)\right| d s \\
& \leqslant \frac{L}{\Gamma(\alpha)} \int_{0}^{t}(t-s)^{\alpha-1}\left|h_{1}(s)-h_{2}(s)\right| e^{-A s} e^{A s} d s \\
& \leqslant \frac{L C_{h_{1}, h_{2}}}{\Gamma(\alpha)} \int_{0}^{t}(t-s)^{\alpha-1} \varphi(s) e^{A s} d s \\
& \leqslant \frac{N L C_{h_{1}}, h_{2}}{\Gamma(\alpha) A} \varphi(t) e^{A t}
\end{aligned}
$$

for all $t \in I$. Therefore, we have

$$
\left|\mathcal{T h} h_{1}(t)-\mathcal{T h} h_{2}(t)\right| e^{-A t} \leqslant \frac{N L C_{h_{1}, h_{2}}}{\Gamma(\alpha) A} \varphi(t)
$$

for all $t \in I$, which means that

$$
\mathrm{d}\left(\mathcal{T} h_{1}, \mathcal{T} h_{2}\right) \leqslant \frac{N L}{\Gamma(\alpha) A} d\left(h_{1}, h_{2}\right)
$$

for all $h_{1}, h_{2} \in S$. 
Thus we conclude that the operator $\mathcal{T}: S \rightarrow S$ defined by (3.4) is strictly contractive. From fundamental theorem of calculus, it is clear that $\mathcal{T} y \in S$ and thus we infer that

$$
\left|\mathcal{T h} h_{1}(t)-h_{1}(t)\right| e^{-A t}<\infty
$$

for any $h_{1} \in S$ and $t \in I$, i.e., $d\left(\mathcal{T h}_{1}, h_{1}\right)<\infty$ for all $h_{1} \in S$. Similarly,

$$
\left|h_{1}(t)-h(t)\right| e^{-A t}<\infty
$$

for all $h \in S$ and $t \in I$, which means that $d\left(h_{1}, h\right)<\infty$ for all $h \in S$, i.e.,

$$
\left\{h \in S: d\left(h_{1}, h\right)<\infty\right\}=S .
$$

Now we have shown that all the conditions of Theorem 2.8 are satisfied with $S^{*}=S$. It follows from (3.3) that

$$
-\varphi(t) \leqslant{ }^{c} D_{a_{+}}^{\alpha} y(x)-f(x, y(x)) \leqslant \varphi(t)
$$

for all $t \in$ I. If we multiply all sides of the above inequality by $(t-s)^{\alpha-1}$ and integrate from 0 to $t$ to get

$$
|\mathcal{T} y(t)-y| \leqslant{ }^{c} I^{\alpha} \varphi(t) \leqslant \frac{1}{\Gamma(\alpha)} \int_{0}^{t}(t-s)^{\alpha-1} \varphi(s) d s \leqslant \frac{1}{\Gamma(\alpha)} \int_{0}^{t}(t-s)^{\alpha-1} \varphi(s) e^{A s} d s \leqslant \frac{N}{A \Gamma(\alpha)} \varphi(t) e^{A t}
$$

for all $t \in I$. Now the inequality above means that

$$
|\mathcal{T} y(t)-y| e^{-A t} \leqslant \frac{N}{A \Gamma(\alpha)} \varphi(t)
$$

i.e.,

$$
d(\mathcal{T} y, y) \leqslant \frac{N}{A \Gamma(\alpha)} .
$$

Therefore, according to Theorem 2.8, there exists a unique solution $y_{0}: I \rightarrow \mathbb{R}$ of differential equation (2.2) satisfying

$$
d\left(y, y_{0}\right) \leqslant \frac{1}{1-\frac{N L}{\Gamma(\alpha) A}} d(\mathcal{T} y, y) \leqslant \frac{\frac{N}{A \Gamma(\alpha)}}{1-\frac{N L}{\Gamma(\alpha) A}} \varphi(t) e^{-A t} .
$$

It follows from the definition of the metric $d\left(y, y_{0}\right)$ that

$$
\left.\mid y(t)-y_{0}(t)\right) \mid e^{-A t} \leqslant \frac{\frac{N}{A \Gamma(\alpha)}}{1-\frac{N L}{\Gamma(\alpha) A}} \varphi(t) e^{-A t},
$$

which implies that

$$
\left.\mid y(t)-y_{0}(t)\right) \mid \leqslant \frac{\frac{N}{A \Gamma(\alpha)}}{1-\frac{N L}{\Gamma(\alpha) A}} \varphi(t)=\frac{N}{\Gamma(\alpha) A-N L} \varphi(t)
$$

for all $t \in I$.

Remark 3.3. Notice that in our analysis we don't assume that $0<\mathrm{KL}<1$ in Theorem 3.2, while it is required in Theorem 3.1 in the article of Wang [32].

Remark 3.4. Notice that in our analysis we drop the assumption (4) of Theorem 3.1 in the article of Wang [32]. (2.2).

In light of Definition 2.7, the following corollary introduces the Hyers-Ulam stability result of equation 
Corollary 3.5. Suppose that $\mathrm{I}=[0, \mathrm{r}]$ is given for fixed real number $\mathrm{r}$. Let $\mathrm{f}: \mathrm{I} \times \mathbb{R} \rightarrow \mathbb{R}$ is continuous function which satisfies (3.2) for all $\mathrm{t} \in \mathrm{I}$, and some positive constant $\mathrm{L}$. Moreover, if $\epsilon \geqslant 0$ and $\mathrm{y}: \mathrm{I} \rightarrow \mathbb{R}$ is a continuous function which satisfies

$$
\left|{ }^{c} D_{a_{+}}^{\alpha} y(x)-f(x, y(x))\right| \leqslant \epsilon
$$

for all $\mathrm{t} \in \mathrm{I}$, then there exists a unique continuous function $\mathrm{y}_{0}: \mathrm{I} \rightarrow \mathbb{R}$ such that

$$
{ }^{c} D_{a_{+}}^{\alpha} y_{0}(x)=f\left(x, y_{0}(x)\right),
$$

and

$$
\left|y(t)-y_{0}(t)\right| \leqslant \frac{N}{\Gamma(\alpha) A-N L} \epsilon
$$

for all $\mathrm{t} \in \mathrm{I}$ and arbitrary constant $\mathrm{A}$ and $\mathrm{N}$ such that the following inequalities

$$
\frac{\mathrm{NL}}{\mathrm{A} \Gamma(\alpha)}<1, \quad \max \left\{1,2^{1-\alpha}\right\}\left(\frac{\alpha+1}{\alpha}\right) \leqslant N e^{A t}
$$

hold.

Proof. We omit the proof because it is a direct application of Theorem 3.2 if we define the function $\varphi(t):=$ $\epsilon$.

\section{Conclusion}

In this article, we studied both the Hyers-Ulam-Rassias and Hyers-Ulam stability of some fractional differential equation with Caputo derivative. In our study, we drop some of the assumptions of some recent known results. In this way, we improve several earlier outcomes. Potential future work could be to investigate the Hyers-Ulam-Rassias and Hyers-Ulam stability for much more complicated fractional differential equations.

\section{References}

[1] M. Akkouchi, Hyers-Ulam-Rassias stability of nonlinear volterra integral equations via a fixed point approach, Acta Univ. Apulensis Math. Inform., 26 (2011), 257-266. 1

[2] C. Alsina, R. Ger, On some inequalities and stability results related to the exponential function, J. Inequal. Appl., 2 (1998), 373-380. 1

[3] T. Aoki, On the stability of the linear transformation in Banach spaces, J. Math. Soc. Japan, 2 (1950), 64-66. 1

[4] J. A. Baker, The stability of certain functional equations, Proc. Amer. Math. Soc., 112 (1991), 729-732. 1

[5] Y. Başc1, A. Mısır, S. Öğrekçi, On the stability problem of differential equations in the sense of Ulam, Results Math., 75 (2020), 13 pages. 1, 3.1

[6] Y. Başc1, S. Öğrekçi, A. Mısır, Hyers-Ulam-Rassias stability for Abel-Riccati type first-order differential equations, Gazi Univ. J. Sci., 32 (2019), 1238-1252. 1

[7] Y. Başc1, S. Öğrekçi, A. Mısır, On Hyers-Ulam stability for fractional differential equations including the new CaputoFabrizio fractional derivative, Mediterr. J. Math., 16 (2019), 14 pages. 1

[8] D. G. Bourgin, Approximately isometric and multiplicative transformations on continuous function rings, Duke Math. J., 16 (1949), 385-397. 1

[9] N. Brillouët-Belluot, J. Brzdęk, K. Ciepliński, On some recent developments in Ulam's type stability, Abstr. Appl. Anal., 2012 (2012), 41 pages. 1

[10] J. Brzdęk, J. Chudziak, Z. Páles, A fixed point approach to stability of functional equations, Nonlinear Anal., 74 (2011), 6728-6732. 1

[11] J. Brzdęk, K. Ciepliński, A fixed point approach to the stability of functional equations in non-archimedean metric spaces, Nonlinear Anal., 74 (2011), 6861-6867. 1

[12] J. Brzdęk, E. El-hady, Z. Leśniak, Fixed-point theorem in classes of function with values in a dq-metric space, J. Fixed Point Theory Appl., 20 (2018), 16 pages. 1

[13] J. Brzdęk, E. El-hady, J. Schwaiger, Investigations on the Hyers-Ulam stability of generalized radical functional equations, Aequationes Math., 94 (2020), 575-593. 1 
[14] K. Ciepliński, Applications of fixed point theorems to the Hyers-Ulam stability of functional equations-a survey, Ann. Funct. Anal., 3 (2012), 151-164. 1

[15] J. B. Diaz, B. Margolis, A fixed point theorem of the alternative for contractions on a generalized complete metric space, Bull. Amer. Math. Soc., 74 (1968), 305-309. 1, 2

[16] G. L. Forti, Hyers-Ulam stability of functional equations in several variables, Aequationes Math., 50 (1995), 143-190. 1

[17] D. H. Hyers, On the stability of the linear functional equation, Proc. Nat. Acad. Sci. U.S.A., 27 (1941), 222-224. 1, 1

[18] D. H. Hyers, G. Isac, T. M. Rassias, Stability of Functional Equations in Several Variables, Birkäuser, Boston, (1998). 1

[19] S.-M. Jung, Hyers-Ulam stability of linear differential equations of first order, Appl. Math. Lett., 17 (2004), 1135-1140. 1

[20] S.-M. Jung, A fixed point approach to the stability of a Volterra integral equation, Fixed Point Theory Appl., 2007 (2007), 9 pages. 3

[21] S.-M. Jung, A fixed point approach to the stability of differential equations $y=F(x, y)$, Bull. Malays. Math. Sci. Soc. (2), 33 (2010), 47-56. 1

[22] M. W. Michalski, Derivatives of noninteger order and their applications, Dissertationes Math. (Rozprawy Mat.), 1993 (1993), 47 pages. 2

[23] M. Obloza, Hyers stability of the linear differential equation, Rocznik Nauk.-Dydakt. Prace Mat., 13 (1993), 259-270. 1

[24] M. Obloza, Connections between Hyers and Lyapunov stability of the ordinary differential equations, Rocznik Nauk.Dydakt. Prace Mat., 14 (1997), 141-146. 1

[25] S. Ögrrekçi, Stability of delay differential equations in the sense of Ulam on unbounded intervals, Int. J. Optim. Control. Theor. Appl. IJOCTA, 9 (2019), 125-131. 1

[26] Z. Páles, Generalized stability of the Cauchy functional equation, Aequationes Math., 56 (1998), 222-232. 1

[27] T. M. Rassias, On the stability of linear mappings in Banach spaces, Proc. Amer. Math. Soc., 72 (1978), 297-300. 1

[28] T. M. Rassias, On the stability of functional equations in Banach spaces, J. Math. Anal. Appl., 251 (2000), 264-284. 1

[29] L. Székelyhidi, Note on a stability theorem, Canad. Math. Bull., 25 (1982), 500-501. 1

[30] J. Tabor, J. Tabor, General stability of functional equations of linear type, J. Math. Anal. Appl., 328 (2007), 192-200. 1

[31] S. M. Ulam, A Collection of Mathematical Problems, Interscience Publisheres, New York-London, (1960). 1

[32] J. R. Wang, L. L. Lv, Y. Zhou, New concepts and results in stability of fractional differential equations, Commun. Nonlinear Sci. Numer. Simul., 17 (2012), 2530-2538. 3.3, 3.4 